\title{
Urine matrix metalloproteinase-7 and risk of kidney disease progression and mortality in type 2 diabetes
}

\author{
Maryam Afkarian ${ }^{\mathrm{a}}$, Leila R Zelnick ${ }^{\mathrm{a}, \mathrm{b}}$, John Ruzinski ${ }^{\mathrm{a}}$, Bryan Kestenbaum ${ }^{\mathrm{a}}$, Jonathan \\ Himmelfarb $^{a}$, lan H de Boera, and Rajnish Mehrotra ${ }^{a}$ \\ aKidney Research Institute and Division of Nephrology, Department of Medicine, University of \\ Washington, Seattle, WA \\ ${ }^{\mathrm{b} D e p a r t m e n t}$ of Biostatistics, University of Washington
}

\section{Abstract}

\begin{abstract}
Aims-The renin-angiotensin-aldosterone system (RAAS), bone morphogenetic protein (BMP) and WNT pathways are dysregulated in diabetic kidney disease (DKD). Urine excretion of angiotensinogen, gremlin-1 and matrix metalloproteinase-7 (MMP-7), components of the RAAS, BMP and WNT pathways, respectively, is increased in DKD. We asked if this increase is associated with subsequent progression to end-stage renal disease (ESRD) or death.
\end{abstract}

Methods-Using time-to-event analyses, we examined the association of baseline urine concentration of these proteins with progression to ESRD or death in a predominantly MexicanAmerican cohort with type 2 diabetes and proteinuric DKD ( $n=141)$.

\begin{abstract}
Results-Progression to ESRD occurred for 38 participants over a median follow-up of 3.0 years; 39 participants died over a median follow-up of 3.6 years. Urine MMP-7 and gremlin-1 were associated with increased risk of ESRD after adjustment for demographic and clinical covariates. Angiotensinogen showed a U-shaped relationship with ESRD, with the middle tertile associated with lowest risk of ESRD. After additional adjustment for glomerular filtration rate and albuminuria, all associations with ESRD lost significance. Only urine MMP-7 was associated with mortality, and this association remained robust in the fully adjusted model with a Hazard ratio of 3.59 (95\% confidence interval 1.31 to 9.85) for highest vs. lowest tertile. Serum MMP-7 was not associated with mortality and did not attenuate the association of urine MMP-7 with mortality (HR 4.03 for highest vs. lowest urine MMP-7 tertile).
\end{abstract}

Conclusions-Among people with type 2 diabetes and proteinuric DKD, urine MMP-7 concentration was strongly associated with subsequent mortality.

\section{Keywords}

Angiotensinogen; diabetic kidney disease; gremlin-1; MMP-7; mortality; Type 2 diabetes

Corresponding Author: Maryam Afkarian, Box 359606, 325 9th Avenue, Seattle, WA 98104, Telephone (206) 685-3168, Fax (206) 685-9399, afkarian@u.washington.ed.

Publisher's Disclaimer: This is a PDF file of an unedited manuscript that has been accepted for publication. As a service to our customers we are providing this early version of the manuscript. The manuscript will undergo copyediting, typesetting, and review of the resulting proof before it is published in its final citable form. Please note that during the production process errors may be discovered which could affect the content, and all legal disclaimers that apply to the journal pertain. 


\section{Introduction}

Diabetic kidney disease (DKD) affects $30-40 \%$ of people with type 2 diabetes ${ }^{1-3}$ and is a major predictor of long-term mortality in this population. ${ }^{4}$ Development of new diagnostic and therapeutic tools for DKD requires a more detailed understanding of the underlying mechanisms.

Evidence from both animal models and human DKD implicates the renin-angiotensinaldosterone system (RAAS), bone morphogenetic protein (BMP) pathway and WNT pathway in pathogenesis of DKD. Angiotensinogen is the sole substrate for renin, the ratelimiting enzyme for activation of the RAAS pathway. Urine angiotensinogen concentration is increased in experimental and human DKD and is highly correlated with renal RAAS activity. ${ }^{5-8}$ BMP pathway activation counters TGF- $\beta$ signaling, while BMP pathway antagonists, such as gremlin-1, block this inhibition. As such, the observed suppression of the BMP pathway activity in experimental and human DKD is thought to contribute to pathogenesis of DKD. ${ }^{9-15}$ WNT pathway activity is increased in animal and human DKD. ${ }^{16-21}$ Matrix metalloproteinase 7 (MMP-7) is the WNT pathway target gene most highly induced in human DKD kidneys. ${ }^{19}$ Urine MMP-7 concentration correlates with renal WNT pathway activity in animal models and is suppressed by WNT pathway antagonists. ${ }^{21}$

We previously reported that urine angiotensinogen, gremlin-1 and MMP-7 were markedly increased in people with type 1 diabetes who had DKD, compared to those without DKD. ${ }^{22}$ Furthermore, treatment with RAAS inhibitors reduced urine angiotensinogen, but not MMP-7 or gremlin-1, in people with DKD. ${ }^{22}$ In this study, we examined whether the increase in urine concentration of these proteins is associated with progression to end-stage renal disease (ESRD) or mortality in people with type 2 diabetes and proteinuric DKD.

\section{Subjects, Materials and Methods}

\subsection{The study population}

We utilized samples from a prospective study of vascular calcification in patients with type 2 diabetes and DKD ${ }^{23-25}$, recruited from two public hospitals in Los Angeles County, California between 2004-2008. Type 2 diabetes was defined as diabetes diagnosed at $\geq$ age 30 and treated with diet or oral hypoglycemic agents for $\geq 6$ months. DKD was defined as a urine protein-to-creatinine ratio $\searrow 0.5 \mathrm{~g} / \mathrm{g}$ at enrollment or the preceding 12 months and $\geq 1$ of the following: (1) typical histologic changes on kidney biopsy, (2) diabetes duration $\geq 5$ years and diabetic retinopathy or (3) diabetes duration $\geq 10$ years without diabetic retinopathy. Patients with a kidney transplant or on dialysis were excluded. All participants provided written informed consent. The study was approved by the Institutional Review Board at Los Angeles Biomedical Research Institute. Links to participant identification were destroyed after the last study contact (2011). Use of de-identified samples from this study was approved as non-human-subjects research by the Human Subjects Division at the University of Washington. 


\subsection{Outcomes}

The participants were re-evaluated 12 and 24 months after the baseline visit or within 3 months of the first dialysis treatment, whichever came first. Participants were followed until the date of death, last study contact or the date of the link with National Death Index (NDI) or the United States Renal Data System (USRDS), whichever was later. ESRD was defined as maintenance dialysis or kidney transplantation. To ascertain progression to ESRD or occurrence of death, study participants or next of kin were contacted every 6 months via phone, letters or home visits. Dates of dialysis initiation and death were additionally determined from links to USRDS (through 12/31/2008) and NDI (through 12/31/2007), respectively.

\subsection{Exposures}

Fasting blood and urine samples were collected at the baseline study visit. Blood samples were stored at room temperature for 30 minutes, then centrifuged for 10 minutes at 1,500x g. Serum was aliquoted and stored at $-80^{\circ} \mathrm{C}$. Spot urine samples were aliquoted and stored at $-80^{\circ} \mathrm{C}$. Angiotensinogen, gremlin- 1 and MMP-7 were measured using commercial immunoassays, as previously described. ${ }^{22}$ Urine samples were either used directly for immunoassays (MMP-7, angiotensinogen) or concentrated 5-fold (gremlin-1) using Amicon $10 \mathrm{kDa}$ ultrafiltration units (Millipore, Billerica, MA). Serum samples were used after being thawed. Angiotensinogen was measured using a quantitative sandwich Enzyme-LinkedImmunosorbent Assay (ELISA) (IBL-America, Minneapolis, MN), with a minimum detection limit of $30 \mathrm{pg} / \mathrm{mL}$, and intra- and inter-assay coefficients of variations (CV's) of 8 and $14 \%$, respectively. ${ }^{22}$ Gremlin-1 was measured using a quantitative ELISA (Uscnk Life Sciences, Wuhan, China), with a minimum detection limit of $60 \mathrm{pg} / \mathrm{mL}$, and intra- and interassay CV's of 13 and 20\%. ${ }^{22}$ MMP-7 was measured using a quantitative ELISA (R \& D Systems, Minneapolis, MN), with a minimum detection limit of $20 \mathrm{pg} / \mathrm{mL}$, and intra- and inter-assay CV's of 5 and $9 \% .^{22}$

\subsection{Covariates}

Demographic data and past medical history were obtained from self-report and medical records. Medication information was obtained by examination of pill bottles. Vital signs were measured in duplicate and averaged, including blood pressure, which was measured using an appropriate-sized cuff on the right arm after 5 minutes of rest. Body mass index (BMI) was calculated by dividing weight $(\mathrm{kg}$ ) by height $(\mathrm{m})$ squared. Laboratory values were measured in fasting blood and urine samples by the clinical laboratory at the HarborUCLA Medical Center. Hemoglobin A1c was measured using HPLC (Toshio Medics, Inc, F,ster City, CA). Serum creatinine was measured using the enzymatic reaction. ${ }^{26}$ Plasma lipids were measured using conventional enzymatic methods, with LDL cholesterol calculated using the Friedewald formula. ${ }^{27}$ Urine albumin was measured using immunoturbidometric assays. ${ }^{28}$ Urine creatinine was measured using the modified Jaffe reaction. ${ }^{29}$ Glomerular filtration rate (GFR) was estimated using the four-variable equation from the Modification of Diet in Renal Disease (MDRD) study. ${ }^{30}$ 


\subsection{Statistical analysis}

Participants were considered at risk for mortality or ESRD from their baseline visit until the first occurrence of the outcome or censoring due to loss to follow-up or the end of available follow-up. Unadjusted incidence rates were calculated by dividing the number of events by person-years of follow-up. The association between concentration of angiotensinogen, gremlin-1 and MMP-7 and ESRD or mortality was assessed per tertile, as well as doubling, of each protein's concentration, normalized to creatinine for urine proteins. Cox regression was used to estimate the relative hazard of ESRD or mortality, adjusting for relevant covariates. The first model included adjustment for demographic covariates; the second model added smoking status, hemoglobin A1c, systolic blood pressure, LDL, and RAAS inhibitor use. The full model added estimated GFR (eGFR) and log-transformed albumin-tocreatinine ratio (ACR), and quadratic effects of each. The nominal significance was a twosided p-value of $\leq 0.05$. All analyses were conducted with R 3.0.2. ${ }^{31}$

\section{Results}

\subsection{Baseline cohort characteristics}

Primary analyses were conducted in a cohort of 141 participants (Table 1) with type 2 diabetes and DKD. The participants had a mean age of 57 years, were mostly Hispanic (73\%) and 55\% male, with a mean (standard deviation, SD) diabetes duration of 14.7 (5.9) years. There was a high prevalence of smoking (55\%), obesity (mean BMI 31.9) and cardiovascular disease (46\%). Mean (SD) for hemoglobin A1c, LDL and HDL were 8.7\% (2.2\%), 110 (43) and 42 (12) mg/dl, respectively. 87\% were treated with anti-hypertensives, including 76\% with RAAS inhibitors, with mean (SD) systolic and diastolic blood pressures of 156 (27) and 78 (13) $\mathrm{mm} \mathrm{Hg}$, respectively. Among participants on RAAS inhibitors, the majority (84\%) were either on angiotensin converting enzyme inhibitors (ACEI) or on a combination of ACEI and angiotensin receptor blockers (ARBs), while $16 \%$ were on ARBs only. Mean (SD) eGFR and ACR were 59 (23) $\mathrm{mL} / \mathrm{min} / 1.73 \mathrm{~m}^{2}$ and $2.5(2.2) \mathrm{g} / \mathrm{g}$, respectively. Serum MMP-7 was measured in a slightly larger group with available serum samples $(\mathrm{n}=151)$. The serum and urine groups were similar in all available demographic and clinical covariates (Supplementary Table 1). Of the 141 participants, only two (2) were treated with vitamin $\mathrm{D}$ receptor agonists.

Subjects with higher urine MMP-7/creatinine concentrations included more females and Hispanics, had longer diabetes duration, higher systolic blood pressure, LDL and statin use, but lower rates of cardiovascular disease. Subjects in the highest and middle MMP-7 tertile had the lowest and highest eGFR, respectively, while ACR increased with MMP-7 tertile. Hemoglobin A1c was comparable in all tertiles (Table 1). Subjects with higher urine angiotensinogen/creatinine concentrations included more females and Hispanics, had longer diabetes duration, higher blood pressure, A1c and ACR, but less smoking, cardiovascular disease and RAAS inhibitor and statin use (Supplementary Table 2). Participants with higher urine gremlin-1/creatinine concentrations included more females and Hispanics, had longer diabetes duration, higher systolic blood pressure, LDL and ACR and lower eGFR (Supplementary Table 3). 
Urine MMP-7 and angiotensinogen correlated more strongly with albuminuria (r 0.52 and 0.45 , respectively) than eGFR ( $\mathrm{r}-0.13$ and -0.09 , respectively). Urine gremlin- 1 correlated moderately with both eGFR and albuminuria ( $\mathrm{r}-0.43$ and 0.48 , respectively) (Figure 1). Urine gremlin-1 and MMP-7 were also strongly correlated ( $\mathrm{r}$ 0.62). Correlations between other urine and serum proteins were weak to moderate ( $\mathrm{r} 0.18$ to 0.35 , Figure 1 ).

\subsection{Urine proteins and risks for ESRD and mortality}

Of the 141 participants at risk, 38 developed ESRD, and 39 died, over a median (IQR) follow-up of 3.0 (1.7-4.6) years for ESRD and 3.6 (2.4-5.5) years for mortality. Eleven of the participants, who developed ESRD, subsequently died during the follow-up. In time-to-event analyses (Table 2), urine gremlin-1/creatinine and MMP-7/creatinine were strongly associated with ESRD in unadjusted models and after adjustment for demographic parameters (model 1) and standard clinical parameters (model 2). However, the associations lost significance after additional adjustment for baseline eGFR and ACR (model 3). Urine angiotensinogen/creatinine had a U-shaped relationship with ESRD rates with the middle tertile showing the lowest rate of ESRD. These associations also lost significance after adjustment for eGFR and ACR. Accounting for the competing risk of death did not alter these findings (Supplementary Table 4).

Among the quantified urine proteins, only MMP-7 was associated with mortality, in unadjusted analyses, after adjustment for demographic and clinical covariates, as well as further adjustment for eGFR and ACR (HR 3.59, 95\% CI 1.31-9.58 for highest vs. lowest tertile) (Table 3). Additional adjustment for diabetes duration, cardiovascular disease, diagnosis of hypertension (in addition to adjustment for systolic blood pressure) and diagnosis of dyslipidemia (in addition to adjustment for LDL) changed the hazard ratios $\mathbf{5 \%}$ and was therefore not included in the fully adjusted model (data not shown).

\subsection{Urine and serum MMP-7 and risks for ESRD and mortality}

Serum MMP-7 correlated moderately with eGFR and ACR ( $\mathrm{r}-0.51$ and 0.53 , respectively), as well as urine MMP-7/creatinine (r 0.46) (Figure 1). In time-to-event analyses (Table 3), serum MMP-7 was significantly associated with ESRD in demographic and clinically adjusted models (models 1 and 2). However, the association lost significance after additional adjustment for eGFR and ACR (model 3). Serum MMP-7 was not associated with mortality in unadjusted or adjusted analyses. Furthermore, adjustment for serum MMP-7 did not attenuate the association between urine MMP-7 and mortality in the fully adjusted model (HR 4.03, 95\% CI 1.4-11.63 for highest vs. lowest urine MMP-7/creatinine tertile after adjustment for demographic and clinical covariates, eGFR, ACR and serum MMP-7 tertile).

\section{Discussion}

In a predominantly Mexican-American cohort with type 2 diabetes, proteinuric DKD and high rates of progression to ESRD and mortality, urine MMP-7 was associated with an increased risk for mortality. This association remained robust to adjustment for standard demographic and clinical covariates, eGFR and albuminuria, as well as adjustment for serum MMP-7. Urine MMP-7 and gremlin-1 were strongly associated with risk of ESRD after 
adjustment for demographic and clinical covariates. However, these associations lost significance after adjustment for eGFR and albuminuria.

Data from animal models strongly implicates the RAAS, BMP and WNT pathways, specifically the pathway components angiotensinogen, gremlin-1 and MMP-7, in DKD pathogenesis. However, there are limited previous data on association of urine angiotensinogen, gremlin-1 and MMP-7 with DKD progression or mortality. Urine angiotensinogen was associated with accelerated GFR loss over a two-year follow-up in a longitudinal study of 49 patients with CKD, including a subset with DKD. ${ }^{5}$ In another study in 234 patients with type 2 diabetes, urine angiotensinogen correlated inversely with annual GFR change over a 9-year follow-up. ${ }^{32}$ Association between urine angiotensinogen and mortality has not been examined to-date. A high ratio of circulating TGF $\beta$ to BMP-7 predicted subsequent renal outcomes in a case-control study nested within the ADVANCE cohort. ${ }^{33}$ However, association of urine gremlin-1 with ESRD or mortality in DKD has not been examined. During preparation of this manuscript, we noted a study reporting no association between urine MMP-7 and progression to ESRD or death in 67 veterans with type 2 diabetes and CKD. ${ }^{34}$

We observed an association between urine MMP-7 and all-cause mortality, which was robust to adjustment for demographic and clinical covariates, including eGFR and albuminuria, suggesting that the association was not confounded by, or mediated through, reduced GFR or albuminuria. Serum MMP-7 may be associated with more than one cause of death. Higher serum MMP-7 has been associated with worse outcomes in several malignancies. ${ }^{35-38}$ In addition, MMP-7 is highly expressed in atheromatous plaques and aneurysmal vessels. ${ }^{39-41}$ Circulating MMP-7 is associated with adverse cardiovascular outcomes in people with atherosclerosis, ${ }^{42}$ presumably reflecting risk for greater plaque rupture. ${ }^{43,44}$ Given the heavy burden of cardiovascular disease and mortality in DKD, we queried whether the observed association between urine MMP-7 and mortality reflected a stronger association between serum MMP-7 and cardiovascular mortality. However, we found no association between serum MMP-7 and mortality. Furthermore, adjusting for serum MMP-7 did not attenuate the association between urine MMP-7 and mortality, suggesting that this association was independent of serum MMP-7 and may have a renal, rather than systemic, origin.

It is not clear why urine MMP-7 expression may be associated with mortality. MMP-7 can cleave binding partners or protein segments of locally sequestered growth factors $\left(\mathrm{TNFa},{ }^{45,46} \mathrm{EGF}^{47} \mathrm{TGF} \beta\right.$ and IGF-1 ${ }^{48}$ ), releasing them from the membrane or extracellular matrix. In addition, MMP-7 regulates apoptosis of several cell types, including vascular smooth muscle cells ${ }^{44}$ and interstitial fibroblasts. ${ }^{49}$ Thus, higher renal MMP-7 may predispose to mortality by raising growth factor concentrations or influencing cell proliferation systemically. Alternatively, a common underlying mechanism may cause both earlier death and higher renal MMP-7 expression. For example, MMP-7 is highly induced by the WNT pathway, whose increased activity is linked to higher mortality in people with ESRD. ${ }^{50}$ MMP-7 is also involved in maintenance of innate mucosal defense against bacteria and is strongly induced by bacterial products. ${ }^{51}$ So another possibility is that a chronically elevated bacterial burden may contribute to a rise both in renal MMP-7 and risk of mortality. 
Identifying the involved pathways would require comprehensive evaluation of each pathway by quantifying concentration of additional protein components.

Our observation of a robust association between urine MMP-7 and mortality contrasts with a recent study not finding evidence for such an association ${ }^{34}$. This discrepancy may be due to the higher power in this study due to our higher event rates (39 vs. 16) and use of time-toevent (vs. logistic) analysis, or the differences in study populations (e.g. race, DKD stage). Furthermore, our study population displayed high rates of progression to ESRD and death, likely due to their high-risk clinical profile at the study baseline. As such, the findings in this study may apply most closely to the subset of patients with DKD who resemble this cohort. Nonetheless, the difference between our findings and the recent reports on association between urine MMP-7 and mortality highlights the need for replication of our findings in other populations.

The association between urine gremlin-1 and MMP-7 with ESRD lost significance after adjustment for eGFR and albuminuria. There are several possible explanations for this finding. First, this study may lack adequate power to detect an association. Secondly, the observed association between urine MMP-7 or gremlin-1 and ESRD may be due to confounding by their association with baseline eGFR or albuminuria. Alternatively, inhibition of the BMP pathway (via increased gremlin-1) and/or activation of the WNT pathway (leading to increased urine MMP-7) may predispose to ESRD by reducing GFR or increasing albuminuria. Interestingly, participants with higher urine gremlin-1 had a lower eGFR (Figure 1), raising the possibility that higher gremlin-1 levels may contribute to reduced GFR, for example by inhibiting the BMP pathway, as supported by preexisting data in animal models. Reduced eGFR predisposes to ESRD. Therefore, adjusting for reduced eGFR (intermediate variable) would eliminate the association between gremlin-1 and ESRD. If so, association between urine gremlin-1 and DKD progression could only be examined in a cohort with normal eGFR at baseline.

We did not find an association between urine angiotensinogen and progression to ESRD, which appears to contrast with prior reports. 5,32 There are several possible explanations for this discrepancy. One reason may be the difference between the studied outcomes (GFR loss vs. ESRD). Another explanation may be that there are differences in study populations. For example, one published study was conducted in a largely non-DKD population, and adjusted for binary forms of eGFR and proteinuria, ${ }^{5}$ while we adjusted for continuous eGFR and ACR, in simple and quadratic forms. Another previous study in subjects with diabetes only described correlations, unadjusted for eGFR or albuminuria. ${ }^{32}$ Moreover, a minority of the participants in these studies were treated with RAAS inhibitors, while the majority (76\%) of participants in our study were using RAAS inhibitors. RAAS inhibitors reduce urine angiotensinogen $5,52-54$ and therefore may modify the association between urine angiotensinogen and DKD progression. The high prevalence of RAAS inhibitor use in our cohort may also explain the U-shaped relation between urine angiotensinogen and risk of ESRD as subjects in the lowest tertile of urine angiotensinogen concentration had highest prevalence of RAAS inhibitor use (83\% vs. $72 \%$, Supplementary Table 2). 
The strengths of this study are use of a clinically relevant population, composed of an ethnic background with high prevalence of DKD and high rates of ESRD and mortality, reliance on hard clinical outcomes of ESRD and mortality and careful adjustment of covariates. The limitations are the modest cohort size, absence of information on adjudicated causes of death, and lack of information on diet and measures of insulin resistance. Furthermore, the individual proteins quantified in this study can participate in multiple pathways. Thus, definite identification of the involved pathways requires future work including a comprehensive assessment of each pathway by quantifying multiple pathway protein components.

In summary, in a largely Mexican-American cohort with type 2 diabetes and proteinuric DKD, urine MMP-7 was associated with mortality after adjustment for demographic and clinical covariates and serum MMP-7, suggesting a possible link between renal WNT pathway activity and mortality.

\section{Supplementary Material}

Refer to Web version on PubMed Central for supplementary material.

\section{Acknowledgments}

M.A. contributed to the study design, wrote and revised the manuscript. L.R.Z. conducted the statistical analysis and contributed to the statistical methods. J.R. performed the experiments. B.K., J.H., I.H.dB. and R.M. reviewed and edited the manuscript. R.M. conducted the original cohort study which contributed samples for studies in this manuscript. All authors read and agreed with the submitted manuscript. M.A. is the guarantor of this work, has had full access to all of the data in this study and takes responsibility for the integrity of the data and the accuracy of the analyses.

Funding: Dr. Afkarian's effort was supported by grant K23DK089017 from the NIDDK and a Norman S. Coplon Extramural Grant from Satellite Healthcare. Dr. de Boer's effort was supported by grants R01DK087726, R01DK087726-S1 and R01DK088762 from the NIDDK. Dr. Mehrotra was supported by grants R01DK95668, and R01DK99165 from the NIH and CER-D2C-1310-07253 from PCORI.

\section{References}

1. KDOQI Clinical Practice Guidelines and Clinical Practice Recommendations for Diabetes and Chronic Kidney Disease. Am J Kidney Dis. 2007; 49:S12-154. [PubMed: 17276798]

2. Hao S, et al. Targeted inhibition of beta-catenin/CBP signaling ameliorates renal interstitial fibrosis. Journal of the American Society of Nephrology: JASN. 2011; 22:1642-1653. [PubMed: 21816937]

3. de Boer IH, et al. Temporal trends in the prevalence of diabetic kidney disease in the United States. Jama. 2011; 305:2532-2539. [PubMed: 21693741]

4. Afkarian M, et al. Kidney disease and increased mortality risk in type 2 diabetes. Journal of the American Society of Nephrology: JASN. 2013; 24:302-308. DOI: 10.1681/ASN.2012070718 [PubMed: 23362314]

5. Yamamoto T, et al. Urinary angiotensinogen as a marker of intrarenal angiotensin II activity associated with deterioration of renal function in patients with chronic kidney disease. Journal of the American Society of Nephrology: JASN. 2007; 18:1558-1565. [PubMed: 17409316]

6. Kobori $\mathrm{H}$, et al. Urinary angiotensinogen as a potential biomarker of severity of chronic kidney diseases. J Am Soc Hypertens. 2008; 2:349-354. [PubMed: 18958182]

7. Kim SS, et al. Clinical implication of urinary tubular markers in the early stage of nephropathy with type 2 diabetic patients. Diabetes Res Clin Pract. 2012; 97:251-257. [PubMed: 22440044]

8. Mills KT, et al. Increased urinary excretion of angiotensinogen is associated with risk of chronic kidney disease. Nephrology, dialysis, transplantation: official publication of the European Dialysis 
and Transplant Association -European Renal Association. 2012; 27:3176-3181. DOI: 10.1093/ndt/ gfs011

9. McMahon R, et al. IHG-2, a mesangial cell gene induced by high glucose, is human gremlin. Regulation by extracellular glucose concentration, cyclic mechanical strain, and transforming growth factor-beta1. J Biol Chem. 2000; 275:9901-9904. [PubMed: 10744662]

10. Wang SN, Lapage J, Hirschberg R. Loss of tubular bone morphogenetic protein-7 in diabetic nephropathy. Journal of the American Society of Nephrology: JASN. 2001; 12:2392-2399. [PubMed: 11675415]

11. Yeh $\mathrm{CH}$, Chang CK, Cheng MF, Lin HJ, Cheng JT. Decrease of bone morphogenetic protein-7 (BMP-7) and its type II receptor (BMP-RII) in kidney of type 1-like diabetic rats. Horm Metab Res. 2009; 41:605-611. [PubMed: 19440953]

12. Dolan V, et al. Expression of gremlin, a bone morphogenetic protein antagonist, in human diabetic nephropathy. Am J Kidney Dis. 2005; 45:1034-1039. [PubMed: 15957132]

13. De Petris L, Hruska KA, Chiechio S, Liapis H. Bone morphogenetic protein-7 delays podocyte injury due to high glucose. Nephrology, dialysis, transplantation: official publication of the European Dialysis and Transplant Association - European Renal Association. 2007; 22:34423450.

14. Walsh DW, et al. Co-regulation of Gremlin and Notch signalling in diabetic nephropathy. Biochim Biophys Acta. 2008; 1782:10-21. [PubMed: 17980714]

15. Turk T, et al. BMP signaling and podocyte markers are decreased in human diabetic nephropathy in association with CTGF overexpression. J Histochem Cytochem. 2009; 57:623-631. [PubMed: 19255250]

16. Kato $\mathrm{H}$, et al. Wnt/beta-catenin pathway in podocytes integrates cell adhesion, differentiation, and survival. J Biol Chem. 2011; 286:26003-26015. [PubMed: 21613219]

17. Rooney B, et al. CTGF/CCN2 activates canonical Wnt signalling in mesangial cells through LRP6: implications for the pathogenesis of diabetic nephropathy. FEBS Lett. 2011; 585:531-538. [PubMed: 21237163]

18. Zhou T, et al. Implication of dysregulation of the canonical wingless-type MMTV integration site (WNT) pathway in diabetic nephropathy. Diabetologia. 2012; 55:255-266. [PubMed: 22016045]

19. Cohen CD, et al. Improved elucidation of biological processes linked to diabetic nephropathy by single probe-based microarray data analysis. PloS one. 2008; 3:e2937. [PubMed: 18698414]

20. Dai C, et al. Wnt/beta-catenin signaling promotes podocyte dysfunction and albuminuria. Journal of the American Society of Nephrology: JASN. 2009; 20:1997-2008. [PubMed: 19628668]

21. He W, et al. Matrix metalloproteinase-7 as a surrogate marker predicts renal Wnt/beta-catenin activity in CKD. Journal of the American Society of Nephrology: JASN. 2012; 23:294-304. [PubMed: 22095947]

22. Afkarian M, et al. Urinary excretion of RAS, BMP, and WNT pathway components in diabetic kidney disease. Physiological reports. 2014; 2(e12010)

23. Mehrotra R, et al. Determinants of coronary artery calcification in diabetics with and without nephropathy. Kidney Int. 2004; 66:2022-2031. DOI: 10.1111/j.1523-1755.2004.00974.x [PubMed: 15496175]

24. Chiu YW, et al. Coronary artery calcification and mortality in diabetic patients with proteinuria. Kidney Int. 2010; 77:1107-1114. DOI: 10.1038/ki.2010.70 [PubMed: 20237457]

25. Chiu YW, et al. Prevalence and prognostic significance of renal artery calcification in patients with diabetes and proteinuria. Clinical journal of the American Society of Nephrology: CJASN. 2010; 5:2093-2100. DOI: 10.2215/CJN.03730410 [PubMed: 20705966]

26. Moss GA, Bondar RJ, Buzzelli DM. Kinetic enzymatic method for determining serum creatinine. Clinical chemistry. 1975; 21:1422-1426. [PubMed: 1157307]

27. Friedewald WT, Levy RI, Fredrickson DS. Estimation of the concentration of low-density lipoprotein cholesterol in plasma, without use of the preparative ultracentrifuge. Clinical chemistry. 1972; 18:499-502. [PubMed: 4337382]

28. Kearney EM, Mount JN, Watts GF, Slavin BM, Kind PR. Simple immunoturbidimetric method for determining urinary albumin at low concentrations using Cobas-Bio centrifugal analyser. Journal of clinical pathology. 1987; 40:465-468. [PubMed: 3584492] 
29. O'Leary N, Pembroke A, Duggan PF. A simplified procedure for eliminating the negative interference of bilirubin in the Jaffe reaction for creatinine. Clinical chemistry. 1992; 38:1749_ 1751. [PubMed: 1526008]

30. Levey AS, et al. A new equation to estimate glomerular filtration rate. Annals of internal medicine. 2009; 150:604-612. [PubMed: 19414839]

31. R_Development_Core_Team. R: A language and environment for statistical computing. 2013

32. Sawaguchi $\mathrm{M}$, et al. Association between urinary angiotensinogen levels and renal and cardiovascular prognoses in patients with type 2 diabetes mellitus. Journal of diabetes investigation. 2012; 3:318-324. [PubMed: 22822420]

33. Wong MG, et al. Circulating bone morphogenetic protein-7 and transforming growth factor-beta1 are better predictors of renal end points in patients with type 2 diabetes mellitus. Kidney Int. 2013; 83:278-284. DOI: 10.1038/ki.2012.383 [PubMed: 23235570]

34. Agarwal R, et al. A prospective study of multiple protein biomarkers to predict progression in diabetic chronic kidney disease. Nephrology, dialysis, transplantation: official publication of the European Dialysis and Transplant Association - European Renal Association. 2014; 29:22932302. DOI: $10.1093 /$ ndt/gfu 255

35. Svatek RS, et al. A multiplexed, particle-based flow cytometric assay identified plasma matrix metalloproteinase-7 to be associated with cancer-related death among patients with bladder cancer. Cancer. 2010; 116:4513-4519. DOI: 10.1002/cncr.25401 [PubMed: 20572047]

36. Malaguarnera G, et al. Serum markers of intrahepatic cholangiocarcinoma. Disease markers. 2013; 34:219-228. DOI: 10.3233/DMA-130964 [PubMed: 23396291]

37. Liu Z, et al. A systematic review and meta-analysis of diagnostic and prognostic serum biomarkers of colorectal cancer. PloS one. 2014; 9:e103910. [PubMed: 25105762]

38. Chang WJ, Du Y, Zhao X, Ma LY, Cao GW. Inflammation-related factors predicting prognosis of gastric cancer. World journal of gastroenterology: WJG. 2014; 20:4586-4596. DOI: 10.3748/ wjg.v20.i16.4586 [PubMed: 24782611]

39. Galis ZS, Sukhova GK, Lark MW, Libby P. Increased expression of matrix metalloproteinases and matrix degrading activity in vulnerable regions of human atherosclerotic plaques. The Journal of clinical investigation. 1994; 94:2493-2503. DOI: 10.1172/JCI117619 [PubMed: 7989608]

40. Thompson RW, Parks WC. Role of matrix metalloproteinases in abdominal aortic aneurysms. Annals of the New York Academy of Sciences. 1996; 800:157-174. [PubMed: 8958991]

41. Halpert I, et al. Matrilysin is expressed by lipid-laden macrophages at sites of potential rupture in atherosclerotic lesions and localizes to areas of versican deposition, a proteoglycan substrate for the enzyme. Proceedings of the National Academy of Sciences of the United States of America. 1996; 93:9748-9753. [PubMed: 8790402]

42. Abbas A, et al. Matrix metalloproteinase 7 is associated with symptomatic lesions and adverse events in patients with carotid atherosclerosis. PloS one. 2014; 9:e84935. [PubMed: 24400123]

43. Johnson JL, George SJ, Newby AC, Jackson CL. Divergent effects of matrix metalloproteinases 3 , 7,9 , and 12 on atherosclerotic plaque stability in mouse brachiocephalic arteries. Proceedings of the National Academy of Sciences of the United States of America. 2005; 102:15575-15580. DOI: 10.1073/pnas.0506201102 [PubMed: 16221765]

44. Williams H, Johnson JL, Jackson CL, White SJ, George SJ. MMP-7 mediates cleavage of Ncadherin and promotes smooth muscle cell apoptosis. Cardiovascular research. 2010; 87:137-146. DOI: $10.1093 / \mathrm{cvr} / \mathrm{cvq} 042$ [PubMed: 20139113]

45. Gearing AJ, et al. Processing of tumour necrosis factor-alpha precursor by metalloproteinases. Nature. 1994; 370:555-557. DOI: 10.1038/370555a0 [PubMed: 8052310]

46. Mohan MJ, et al. The tumor necrosis factor-alpha converting enzyme (TACE): a unique metalloproteinase with highly defined substrate selectivity. Biochemistry. 2002; 41:9462-9469. [PubMed: 12135369]

47. Yu WH, Woessner JF Jr, McNeish JD, Stamenkovic I. CD44 anchors the assembly of matrilysin/ MMP-7 with heparin-binding epidermal growth factor precursor and ErbB4 and regulates female reproductive organ remodeling. Genes \& development. 2002; 16:307-323. DOI: 10.1101/gad. 925702 [PubMed: 11825873] 
48. Visse R, Nagase H. Matrix metalloproteinases and tissue inhibitors of metalloproteinases: structure, function, and biochemistry. Circulation research. 2003; 92:827-839. DOI: 10.1161/01.RES.0000070112.80711.3D [PubMed: 12730128]

49. Zhou D, Tan RJ, Zhou L, Li Y, Liu Y. Kidney tubular beta-catenin signaling controls interstitial fibroblast fate via epithelial-mesenchymal communication. Scientific reports. 2013; 3:1878. [PubMed: 23698793]

50. Viaene L, et al. Sclerostin: another bone-related protein related to all-cause mortality in haemodialysis? Nephrology, dialysis, transplantation: official publication of the European Dialysis and Transplant Association -European Renal Association. 2013; 28:3024-3030. DOI: 10.1093/ndt/ gft039

51. Burke B. The role of matrix metalloproteinase 7 in innate immunity. Immunobiology. 2004; 209:51-56. DOI: 10.1016/j.imbio.2004.04.005 [PubMed: 15481140]

52. Ogawa S, et al. Angiotensin II Type 1 Receptor Blockers Reduce Urinary Angiotensinogen Excretion and the Levels of Urinary Markers of Oxidative Stress and Inflammation in Patients with Type 2 Diabetic Nephropathy. Biomark Insights. 2009; 4:97-102. [PubMed: 19652765]

53. Urushihara M, Kondo S, Kagami S, Kobori H. Urinary angiotensinogen accurately reflects intrarenal Renin-Angiotensin system activity. Am J Nephrol. 2010; 31:318-325. [PubMed: 20160435]

54. Jang HR, et al. Potential role of urinary angiotensinogen in predicting antiproteinuric effects of angiotensin receptor blocker in non-diabetic chronic kidney disease patients: a preliminary report. Postgrad Med J. 2012; 88:210-216. [PubMed: 22357774] 

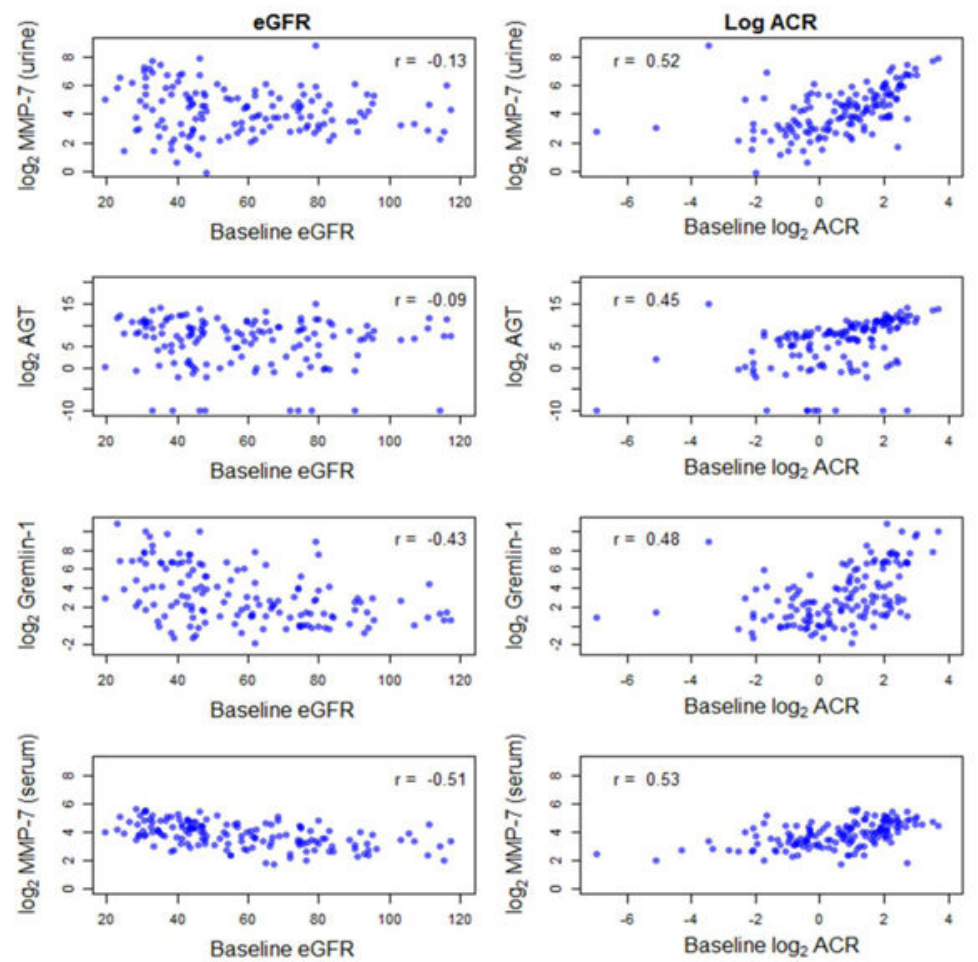

\begin{tabular}{|l|c|c|c|c|}
\hline & $\begin{array}{c}\log _{2} \text { Urine } \\
\text { MMP-7/Cr }\end{array}$ & $\begin{array}{c}\log _{2} \text { Urine } \\
\text { angiotensinogen/Cr }\end{array}$ & $\begin{array}{c}\log _{2} \text { Urine } \\
\text { Gremlin1/Cr }\end{array}$ & $\begin{array}{c}\log _{2} \text { Serum } \\
\text { MMP-7 }\end{array}$ \\
\hline $\log _{2}$ Urine MMP-7/Cr & 1 & 0.27 & 0.62 & 0.46 \\
\hline $\log _{2}$ Urine AGT/Cr & & 1 & 0.35 & 0.18 \\
\hline $\log _{2}$ Urine Gremlin1/Cr & & & 1 & 0.5 \\
\hline $\log _{2}$ Serum MMP-7 & & & & 1 \\
\hline
\end{tabular}

Figure 1.

Correlation between estimated GFR, ACR and creatinine-adjusted urine angiotensinogen, MMP-7, gremlin-1 and serum MMP-7. 
Table 1

Participant characteristics by tertiles of urine MMP-7/creatinine

\begin{tabular}{|c|c|c|c|c|}
\hline Participant characteristic & All & Low $(\leq 10.2 \mu \mathrm{g} / \mathrm{g})$ & $\underset{\leq 32 \mu \mathrm{g} / \mathrm{g})}{\operatorname{Medium}}(10.2<$ and & $\operatorname{High}(>32 \mu \mathrm{g} / \mathrm{g})$ \\
\hline Number & 141 & 47 & 47 & 47 \\
\hline Age (years) & $57(8)$ & $57(7)$ & $57(8)$ & $57(8)$ \\
\hline Female & $63(45)$ & $17(36)$ & $22(47)$ & $24(51)$ \\
\hline \multicolumn{5}{|l|}{ Race/ethnicity } \\
\hline Caucasian & $21(15)$ & $9(19)$ & $7(15)$ & $5(11)$ \\
\hline African-American & $12(9)$ & $8(17)$ & $2(4)$ & $2(4)$ \\
\hline Hispanic & $103(73)$ & $26(55)$ & $38(81)$ & $39(83)$ \\
\hline Other & $5(4)$ & $4(9)$ & $0(0)$ & $1(2)$ \\
\hline \multicolumn{5}{|l|}{ Smoking } \\
\hline Current & $21(15)$ & $7(15)$ & $8(17)$ & $6(13)$ \\
\hline Past & $57(40)$ & $19(40)$ & $18(38)$ & $20(43)$ \\
\hline Diabetes duration (years) & $14.7(5.9)$ & $13.9(4.8)$ & $14.2(6)$ & $15.9(6.5)$ \\
\hline Prevalent cardiovascular disease & $65(46)$ & $24(51)$ & $23(49)$ & $18(38)$ \\
\hline Body mass index $\left(\mathrm{kg} / \mathrm{m}^{2}\right)$ & $31.9(8.7)$ & $33.5(9.5)$ & $31.8(8.8)$ & $30.5(7.8)$ \\
\hline Use of any anti-hypertensives & $123(87)$ & $44(94)$ & $39(83)$ & $40(85)$ \\
\hline $\begin{array}{l}\text { Use of RAAS inhibitors ( } 73 \% \text { on ACEI; } 11 \% \text { on ACEI } \\
\text { +ARB; } 16 \% \text { on ARB) }\end{array}$ & $107(76)$ & $38(81)$ & $33(70)$ & $36(77)$ \\
\hline Use of HMG-CoA reductase inhibitors & $89(63)$ & $27(57)$ & $28(60)$ & $34(72)$ \\
\hline Systolic blood pressure $(\mathrm{mmHg})$ & $156(27)$ & $148(26)$ & $160(25)$ & $160(29)$ \\
\hline Diastolic blood pressure (mmHg) & $78(13)$ & $78(15)$ & $79(12)$ & $76(11)$ \\
\hline Hemoglobin A1c (\%) & $8.7(2.2)$ & $8.6(2.3)$ & $8.9(2.1)$ & $8.6(2.3)$ \\
\hline $\mathrm{LDL}(\mathrm{mg} / \mathrm{dL})$ & $110(43)$ & $104(41)$ & $112(34)$ & $116(52)$ \\
\hline $\mathrm{HDL}(\mathrm{mg} / \mathrm{dL})$ & $42(12)$ & $43(13)$ & $41(11)$ & $43(12)$ \\
\hline eGFR $\left(\mathrm{mL} / \mathrm{min} / 1.73 \mathrm{~m}^{2}\right)$, mean & $59(23)$ & $60(25)$ & $65(22)$ & $51(22)$ \\
\hline eGFR $\left(\mathrm{mL} / \mathrm{min} / 1.73 \mathrm{~m}^{2}\right)$, median & $55(41-75)$ & $54(41-78)$ & $65(47-78)$ & $45(34-64)$ \\
\hline ACR $(g / g)$, mean & $2.5(2.2)$ & $1(0.9)$ & $2.3(1.3)$ & $4.1(2.7)$ \\
\hline ACR $(g / g)$, median & $1.9(0.8-3.9)$ & $0.7(0.4-1.1)$ & $2.1(1.4-3.2)$ & $4.1(2.1-5.5)$ \\
\hline Urine Angiotensinogen $/ \mathrm{Cr}(\mu \mathrm{g} / \mathrm{g})$, median & $200(6-1145)$ & $41(2-171)$ & $213(36-975)$ & $1568(204-3322)$ \\
\hline Urine Gremlin- $1 / \mathrm{Cr}(\mu \mathrm{g} / \mathrm{g})$, median & $6(2-39)$ & $2(1-6)$ & $6(2-20)$ & $92(6-186)$ \\
\hline Urine MMP-7/Cr $(\mu \mathrm{g} / \mathrm{g})$, median & $19(8-42)$ & $7(5-8)$ & $19(14-24)$ & $60(42-101)$ \\
\hline
\end{tabular}

Data is displayed as mean (standard deviation, SD) for continuous covariates, number (percent) for categorical covariates and median (interquartile range, IQR) where noted. RAAS: renin angiotensin saldosterone ystem; ACEI: angiotensin converting enzyme inhibitors; ARB: angiotensin receptor blockers; ACR: albumin to creatinine ratio; eGFR: estimated glomerular filtration rate. 


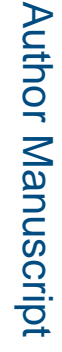

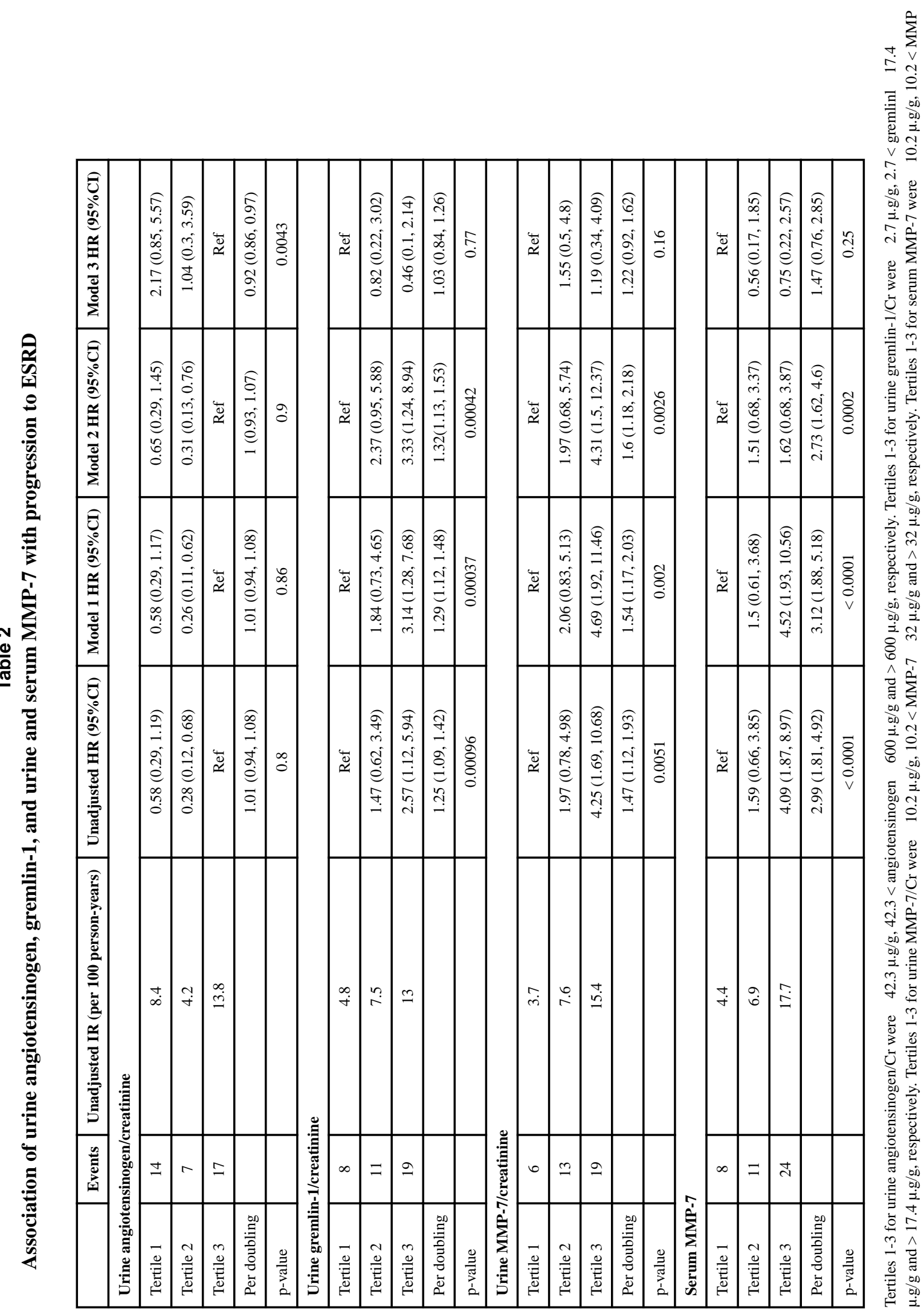

J Diabetes Complications. Author manuscript; available in PMC 2017 April 12. 


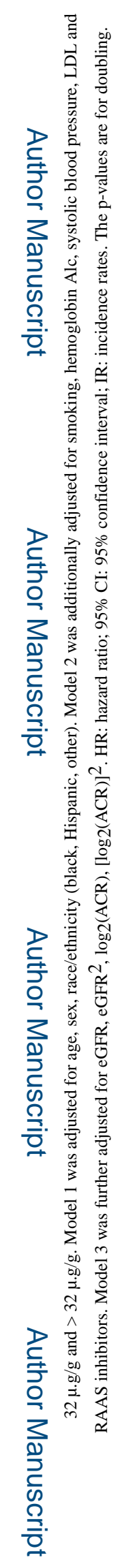




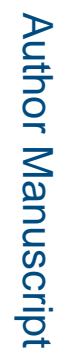

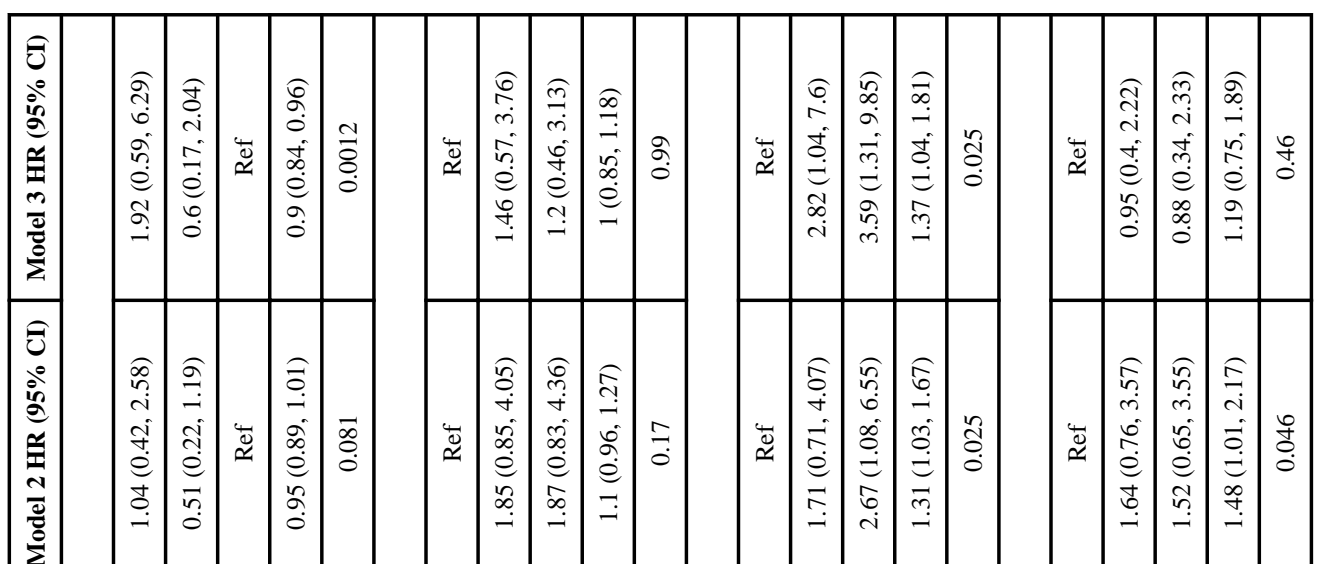



竞

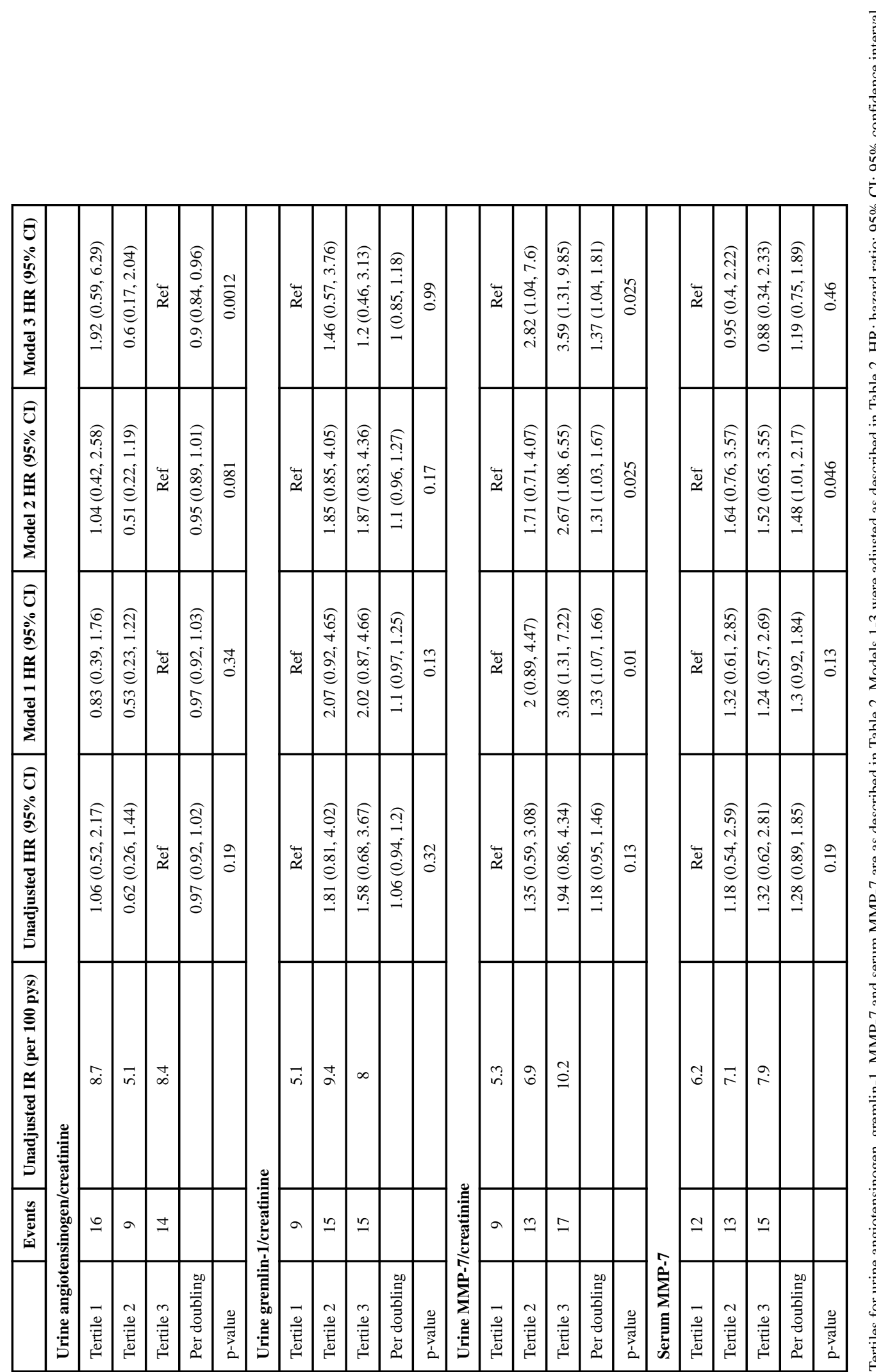

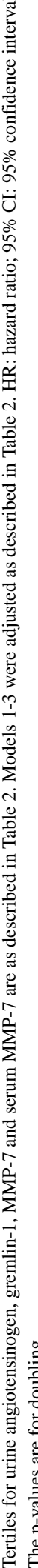

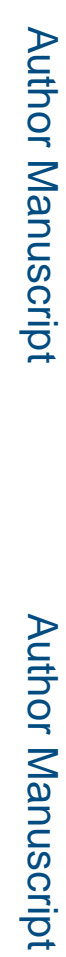

J Diabetes Complications. Author manuscript; available in PMC 2017 April 12. 